\title{
Estilos de liderazgo de los directivos escolares costarricenses: transformando las organizaciones educativas
}

\author{
[............................................... \\ José Antonio García-Martínez* \\ Virginia Cerdas-Montano** \\ * Docente e investigador en la División de Educación para el Trabajo, en el Centro de Investigación y \\ Docencia en Educación de la Universidad Nacional, Heredia, Costa Rica \\ iD orcid.org/0000-0003-0709-0814 \\ jose.garcia.martinez@una.cr \\ ** Docente e investigadora en la División de Educación para el Trabajo, en el Centro de Investigación y \\ Docencia en Educación de la Universidad Nacional, Heredia, Costa Rica. \\ (iD) orcid.org/0000-0003-1705-4630 \\ norma.cerdas.montano@una.cr
}

Recibido: 24 de abril del 2019 Corregido: 09 de julio del 2019 Aceptado: 24 de julio del 2019

\begin{abstract}
Resumen
El estudio del liderazgo se ha tornado relevante en el desarrollo de las organizaciones en general, y en el contexto educativo en particular. La investigación muestra como objetivo analizar los estilos de liderazgo de directivos de centros educativos costarricenses. Se propone una metodología cuantitativa, con un diseño ex post facto y transversal, aplicando el cuestionario Multifactor Leadership Questionnaire (MLQ) a una muestra probabilística estratificada de 125 directivos. Los hallazgos muestran un estilo de liderazgo transformacional predominante, seguido del estilo transaccional, resaltando la baja frecuencia del estilo laissez -faire. Se recomienda que el liderazgo transformacional tome como eje dinamizador la dimensión pedagógica, la cual caracteriza el trabajo que se ejecuta en las organizaciones educativas, por tanto, el fortalecimiento de un liderazgo desde lo pedagógico direccionado hacia un trabajo colaborativo que favorezca procesos identitarios, ya que el logro de metas conjuntas es sustantivo para la calidad de la oferta educativa.
\end{abstract}

Palabras clave: Liderazgo; liderazgo transformacional; centros educativos; estilos de liderazgo; educación.

\section{Abstract \\ Leadership styles of Costa Rican school directives: transforming educational organizations}

The study of leadership has risen importance in the development of organizations in general, particularly in the educational context. The present investigation has the objective of analizing the styles of leadership directives of Costa Ricans schools. It is proposed the quantitive methology, with an expost facto and transversal desing, implementing the questionnaire Multifactor Leadership Questionnaire (MLQ) applied to a stratified probabilistic sample of 125 school principals. The findings show a predominant transformational leadership style, followed by the transactional style, highlighting the low frequency of the laissez-faire style. It is recommended that the transformational leadership take as dynamic axis the pedagogical dimension, which characterizes the work executed in the educational organizations, consecuently, the strengthening of a leadership from the pedagogical axis to a collaborative work that favors identity process, resulting in the achievement of joint goals is substantive for the quality of the educational offer.

Key words: Leadership; transformational leadership; educational centers; leadership styles; education. 


\section{INTRODUCCIÓN}

El liderazgo es uno de los temas que ha tomado un lugar relevante en el desarrollo de las organizaciones en las últimas décadas (Delgado, 2005; Trujillo, López \& Lorenzo, 2016; Buitrago, Escobar \& Terraza, 2017; Marticorena, 2018). Las posibilidades de abordaje desde diferentes ámbitos promueven que un acercamiento conceptual al liderazgo se torne complejo. Sin embargo, los estudios coinciden en que el liderazgo en las organizaciones educativas es caracterizado por su comportamiento e influencia en los equipos de trabajo para alcanzar metas comunes, acuñando valores y principios desde un marco ético que favorece los procesos de aprendizaje para potenciar los talentos de los miembros de la comunidad educativa (Garbanzo \& Orozco, 2007; Montero, Fernández, Barquero \& Martínez, 2017).

Los hallazgos en torno al tema de liderazgo, tanto en el ámbito nacional como internacional, hacen contribuciones sustantivas que permiten una comprensión global de su importancia en el desarrollo de las organizaciones; no obstante, requiere de un análisis particular para cada contexto en donde se generan procesos educativos, ya que convergen múltiples factores actitudinales, conceptuales, de personalidad, culturales, entre otros que afectan el desempeño (Cerdas, Torres \& García, 2016).

Asimismo, existe una estrecha relación entre el liderazgo y la cultura organizacional que se desarrolla en los centros educativos, la cual a su vez se vincula con la efectividad de logros de la organización (Stilman, 2001; Mendoza \& Ortiz, 2006; Alatorre, 2016); por tanto, los estudios sobre el tema de liderazgo se tornan de relevancia para las organizaciones que desean efectivizar los logros y apostar por entornos laborales colaborativos. Lo anterior implica la necesidad de un análisis exhaustivo relacionado con los elementos variados que confluyen para definir un estilo de liderazgo.

En el ámbito costarricense, algunos estudios refieren a la necesidad del desarrollo del liderazgo para la gestión de centros educativos (Garbanzo \& Orozco, 2007; Montero et al., 2017; García, Cerdas \& Torres, 2018), enfatizando en la capacidad de direccionar equipos de trabajo, efectividad de logros, satisfacción de las demandas y necesidades a través de la oferta educativa pública que se brinda en las instituciones.

Aunado a lo anterior, el Sexto Informe del Estado de la Educación (2017) refiere al mejoramiento de la calidad docente como una prioridad de la política educativa, ya que este colectivo es la clave principal para mejorar las ofertas curriculares desarrolladas en los centros. Los docentes asignan un valor alto al papel que juegan el centro educativo y las familias para el éxito escolar, lo que direcciona los esfuerzos hacia prácticas que favorezcan un trabajo colaborativo que permita el mejoramiento de la calidad de la educación.

En este sentido, el rol de la persona directiva trasciende el quehacer administrativo, constituyéndose en un promotor de cambios, un estratega que busca condiciones para la viabilidad del proyecto educativo, un líder comunitario que escucha las necesidades del contexto a la vez que plantea alternativas de solución colegiadas con el equipo docente y administrativo (García et. al, 2018). Por tanto, asume al centro educativo como una comunidad de aprendizaje, en donde cada uno de sus miembros aporta esfuerzos conjuntos para una cultura institucional que apuesta por una oferta educativa de calidad (Zenteno \& Álvarez, 2016).

A nivel internacional, el liderazgo ha sido motivo de múltiples investigaciones tanto en el ámbito de las organizaciones en general (Loaiza, 2017; García-Solarte, 2015; Hernández, Tobón, \& Vázquez, 2015) como en las instituciones educativas en particular (Torrecilla, 2016; Corbella, 2016; Sánchez, 2015; Botía, Rodríguez \& García-Garnica, 2017), siendo uno de los instrumentos más utilizados para la medición del estilo de liderazgo de las organizaciones el Multifactor Leadership Questionnaire (MLQ), (Molero, Recio \& Cuadrado, 2010). 
En el ámbito educativo se encuentran algunas aplicaciones del instrumento (Manning, 2016; Bagheri \& Sohrabi, 2015; Bryant, Butcher \& O'Connor, 2016; Allen, Grigsby \& Peters, 2015) y específicamente en Costa Rica no se encuentra ninguna referencia de su utilización. De lo anterior se deriva parte de la importancia de esta investigación, con el objetivo de analizar el estilo de liderazgo predominante en los directivos de los centros educativos costarricenses.

\section{Marco teórico}

El acercamiento conceptual al término de liderazgo en el ámbito educativo refiere a la capacidad de direccionar un trabajo colaborativo que posee como eje central lo pedagógico, desde procesos compartidos y distributivos que permitan el alcance de metas conjuntas para el beneficio directo del estudiantado (Botía, 2015).

Ante los desafíos que la educación presenta, surge el liderazgo como una alternativa que puede apoyar de manera efectiva los procesos de cambio que se requieren (Ruiz, 2013). Por consiguiente, uno de los desafíos permanentes para los equipos directivos de centros educativos es sostener procesos de mejora que permitan alcanzar indicadores de calidad y favorezcan estilos de liderazgos asociados con el trabajo colaborativo.

En este estudio, se analizan tres estilos de liderazgo de los directivos escolares; transformacional, transaccional y laissez faire. Para cada uno de ellos se establecen algunas dimensiones que caracterizan las prácticas cotidianas que se desarrollan en los centros educativos, asociadas a un liderazgo en particular.

\section{El liderazgo transformacional}

En el estilo de liderazgo transformacional prevalece un reconocimiento al directivo escolar como autoridad que gestiona el centro educativo, así se promueve el desarrollo de la organización con un vínculo fuerte de confianza de sus colaboradores. Es capaz de trascender sus propios intereses por los de la organización, por lo que muestra una imagen de autoridad moral y ética aceptable, caracterizada por elevar los deseos de superación del equipo docente hacia el trabajo colaborativo (Hermosilla, Amutio, Costa \& Páez, 2016).

Entre los pioneros con mayores aportes al liderazgo transformacional se destacan James Mac Gregor Burns y Bernard Bass, quienes caracterizan los roles ejercidos en la organización desde los equipos directivos y que tiene relación directa con las metas, aspiraciones y capacidades. Además, destaca aspectos representativos del centro educativo como su cultura, programas, políticas, recursos, entre otros que se conjugan con características emocionales de la persona, lo cual potencializa el desarrollo de sus docentes para el alcance de logros comunes a través del trabajo colaborativo (Leithwood, 2009).

También se identifican características relevantes en el liderazgo transformacional en relación con la capacidad de superación de intereses propios del directivo al anteponer los de la organización. Además, de generar un sentido de confianza entre sus docentes, promoviendo la autosuperación y por ende el desarrollo de la organización.

Entre las cuatro dimensiones insertas en el liderazgo transformacional (Avolio \& Bass, 2004), se encuentra la estimulación intelectual la cual plantea un apoyo constante para los colaboradores en el desarrollo de pensamiento creador que permita buscar alternativas innovadoras a los problemas a los que se enfrenten.

Una segunda dimensión es la influencia idealizada (conducta y atributos) la cual establece que los líderes poseen una influencia positiva, son admirados y gozan de la confianza y el respeto de sus colaboradores. 
En algunos casos sirven de inspiración para los mismos y pueden ser ejemplo de quienes desean seguir como modelos de liderazgo. A su vez, se deriva en dos dimensiones: atribuida y conducta (Molero et al., 2010) la diferencia básica es que la primera centra su atención en la influencia que ejerce en sus colaboradores, mientras en la segunda se centra en conductas específicas que ejerce el líder para mantener coherencia ética entre el discurso y sus acciones.

La tercera dimensión es la motivación inspiracional, la cual se centra en la capacidad de los líderes para motivar y alentar a sus colaboradores, se facilita con ello el trabajo en equipo relacionado con la construcción de una visión clara y compartida del rumbo de la organización. Por último, se contempla la consideración individualizada, la cual presta especial atención a las necesidades de cada colaborador y permite brindar seguimiento a su crecimiento y desarrollo de habilidades.

\section{Liderazgo transaccional}

El liderazgo transaccional se caracteriza principalmente en un intercambio de costo y beneficio (Avolio \& Bass, 2004); se centra en la persona y se gestiona desde un proceso planificado e intencionado, con una estructura rígida que pretende eliminar la incertidumbre, con una relación lineal entre las autoridades y los colaboradores (Contreras \& Barbosa, 2013).

Las dimensiones que responden a este estilo son por un lado la recompensa contingente, la cual refiere específicamente al reconocimiento que se le da al colaborador en relación con el trabajo realizado, es decir un premio por el logro obtenido. De antemano se requiere una clara asignación de roles y responsabilidades, pues existe una planificación previa que retoma todos los detalles esperados como resultados. Por otro lado, la dirección por excepción pasiva se refiere a que el líder solo interviene cuando existen problemas serios y los logros no son los esperados. En relación con la dirección por excepción activa se centra en una intervención del líder en caso de que haya alguna situación de descontrol; si se presentan errores evidentes y se requiere llamar la atención sobre lo ocurrido para corregir la situación y llegar a los resultados esperados.

La diferencia entre la dirección por excepción activa y la pasiva es que en la primera existe un monitoreo activo que llama la atención en los errores en los que se incurre por parte de los miembros del equipo y en la segunda se presenta una intervención cuando el error ya se ha cometido.

\section{Laissez faire}

El estilo de liderazgo laissez faire apunta hacia una participación casi nula del líder en los procesos que se desarrollan en la organización, evitando todo tipo de influencia hacia los procesos o hacia las personas, además de la carencia definida de metas u objetivos que marcan la ruta para el equipo de trabajo (Vílchez, Alberola, Monte \& Ferraz, 2019).

Según Avolio y Bass (2004), también se denomina un no liderazgo y se centra en una abstinencia a guiar cualquier proceso o de dar información más allá de la que solicitan expresamente los miembros de la organización, se caracteriza por la ausencia de una planificación que deriva en falta de claridad de roles y de responsabilidades que se asumen en el centro educativo.

\section{Efectividad, esfuerzo extra y satisfacción}

Además de las dimensiones descritas para cada estilo de liderazgo, autores como Bass y Avolio (1994) plantean otras variables como el esfuerzo extra, la satisfacción y la efectividad como aspectos importantes de análisis para determinar los estilos de liderazgo. La efectividad se plantea en términos principalmente asociados al rol de representatividad que desarrolla el directivo ante las autoridades y ante los requerimientos administrativos. El esfuerzo extra refiere a la influencia que ejerce el directivo en su 
equipo, en relación con el cumplimento de sus tareas y a la voluntad de desempeñarse de manera que pueda lograr sus metas. Finalmente, la satisfacción se liga a métodos de liderazgo agradables para su equipo de trabajo y a la satisfacción que pueda provocar en los miembros.

\section{Liderazgo pedagógico}

En el caso específico de este estudio la población de interés ejerce su liderazgo precisamente en instituciones educativas, por lo que se considera conveniente articular el liderazgo pedagógico en este análisis.

Para dinamizar procesos en el ámbito educativo, se requiere un liderazgo que direccione sus esfuerzos hacia una misión y visión que tenga como eje central la formación de la persona. Los procesos de enseñanza y aprendizaje constituyen un elemento diferenciador para el liderazgo en los centros educativos, a su vez demanda accionar vínculos entre instituciones educativas y la sociedad (Macías \& Ramírez, 2015). El líder pedagógico está en capacidad de maximizar las metas del centro con miras al mejoramiento, favoreciendo condiciones para que todos sus miembros aprendan de manera continua y colaborativa (Botía et al., 2017).

Macías y Ramírez (2015) refieren que el liderazgo pedagógico se fortalece cuando se logra que los miembros del centro educativo hagan un trabajo colaborativo y son capaces de innovar, aportar conocimientos y nuevas estrategias para el abordaje metodológico y favorecer el crecimiento en valores. Esto permitiría que el cuerpo docente, administrativo, estudiantil y las familias que intervienen como agentes de cambio en los procesos de formación se incorporen a procesos colegiados, además los esfuerzos extras realizados tendrán significado y satisfacción en el tanto se puedan concretar metas y objetivos trazados.

En este sentido, Horn y Marfán (2010) mencionan que el liderazgo pedagógico posee repercusiones directas sobre el aprendizaje de los estudiantes; por ello se considera sustantivo articular estas dimensiones desde las cuales se analizan las prácticas de liderazgo sin perder de vista que responden a un ámbito educativo.

\section{METODOLOGÍA}

La metodología aplicada es de carácter empírico-analítico, sigue un proceso deductivo y un diseño ex post facto (Hernández, Fernández \& Baptista, 2010). Desde este enfoque cuantitativo, la recolección de datos como parte del método operativo se realizó de manera transversal (Hernández et al., 2010) y con un alcance descriptivo.

\section{Población y muestra}

La población queda compuesta por el colectivo de directores de centros educativos de la Dirección Regional de Educación (DRE) de Heredia.

La muestra fue seleccionada de manera probabilística y estratificada (Hernández et. al, 2010), considerando cada uno de los siete circuitos que componen la DRE como estratos. La representatividad queda de manifiesto en la tabla 1, donde puede comprobarse que el porcentaje de la muestra es muy similar al de la población.

La muestra asciende a $n=125$ personas encuestadas, para su cálculo se utilizó la formulación para poblaciones finitas propuesta por Arnal, del Rincón y Latorre (1992). Teniendo en cuenta la población total 
TABLA 1

Composición de la muestra por circuitos de la DRE de Heredia, frecuencias y porcentajes de la población y la muestra seleccionada

\begin{tabular}{|c|c|c|c|c|}
\hline \multirow{2}{*}{ Circuitos } & \multicolumn{2}{|c|}{ Población } & \multicolumn{2}{|c|}{ Muestra } \\
\hline & Frecuencia & Porcentaje & Frecuencia & Porcentaje \\
\hline 01 & 31 & 14,5 & 21 & 17,5 \\
\hline 02 & 30 & 14,0 & 20 & 16,7 \\
\hline 03 & 24 & 11,2 & 18 & 15,0 \\
\hline 04 & 42 & 19,6 & 16 & 13,3 \\
\hline 05 & 29 & 13,6 & 14 & 11,7 \\
\hline 06 & 30 & 14,0 & 17 & 14,2 \\
\hline 07 & 28 & 13,1 & 19 & 15,8 \\
\hline TOTAL & 214 & 100,0 & 125 & 104,2 \\
\hline
\end{tabular}

Fuente: Elaboración propia con base en datos ofrecidos por la supervisión de la Dirección Regional de Educación de Heredia.

de directores ( $\mathrm{N}=214)$; un nivel de confianza $\geq 95 \%$, error $\leq 5 \%$ y con distribución estimada de $50 \%$ de las respuestas, obteniendo del cálculo el número de casos mínimo requerido es de 105, inferior al número final de la muestra. Cabe destacar que la muestra final cumple con dos restricciones: a) haber cumplimentado al menos $75 \%$ del instrumento y b) llevar laborando al menos un año en el puesto de dirección de la actual institución.

La edad del colectivo de la muestra oscila entre los 32 y los 58 años (Media=24; DS=5,9). Del grupo participante, 44 son hombres $(35,2 \%)$, mientras 81 son mujeres $(64,8 \%)$. En cuanto a la distribución por grado, 2 personas (1,6\%) tienen bachillerato, $24(19,2 \%)$ licenciatura, $96(76,8 \%)$ tienen una maestría y los restantes $3(2,4 \%)$ poseen un doctorado.

\section{Técnicas de recolección de datos}

La técnica de recolección de datos utilizada es la encuesta, y como instrumento un cuestionario compuesto por varios bloques de preguntas cerradas, el primero con preguntas socio demográficas, un segundo bloque sobre educación y la formación previa en liderazgo y por último la escala tipo Likert denominada Multifactor Leadership Questionnaire (MLQ 5X) de Bass y Avolio, (1994), la cual a través de 45 ítems (Tabla 2) con cinco opciones de respuesta desde 1 (nunca) hasta 5 (siempre), mide el liderazgo transformacional formado por 5 sub-escalas, el liderazgo transaccional con 3 sub-escalas, el laissez faire a través de una escala, además de las subescalas efectividad, satisfacción y esfuerzo extra.

La recogida de datos se llevó a cabo en coordinación con la supervisión de cada uno de los circuitos de la DRE de Heredia, se aprovecharon las reuniones mensuales. Se realizó una prueba piloto a diez directores con características similares a la muestra final, y se obtuvieron insumos en cuanto a la adaptación al contexto costarricense de algunas de las afirmaciones de la escala.

Por último, se realizó la prueba de consistencia interna Alfa de Cronbach, se obtuvo un coeficiente de 0,74 en el orden de estudios similares (Avolio, Bass \& Jung, 1999; Antonakis, Avolio \& Sivasubramaniam, 2003) y considerados por la teoría como fiables. 
TABLA 2

Definición instrumental del cuestionario MLQ 5X

\begin{tabular}{llc} 
Tipo de Liderazgo & \multicolumn{1}{c}{ Sub escala } & Ítemes \\
Transformacional & Estimulación intelectual & $2,8,30$ y 32 \\
& Influencia idealizada (conducta) & $6,14,23$, y 24 \\
& Influencia idealizada (atributos) & $10,18,21$ y 25 \\
& Motivación por inspiración & $9,13,26$ y 36 \\
Transaccional & Consideraciones individuales & $15,19,29$ y 31 \\
& Premio por contingencia & $1,11,16$ y 35 \\
Laissez-Faire & Gerencia por excepción pasiva & $3,12,17$, y 20 \\
& Gerencia por excepción activa & $4,22,24$ y 27 \\
& & $5,7,28$ y 33 \\
& Efectividad & $37,40,43$ y 45 \\
& Satisfacción & 38 Y 41
\end{tabular}

Fuente: Elaboración propia.

\section{RESULTADOS}

A continuación, se presentan algunos resultados descriptivos de la muestra en relación con los centros educativos de las personas directivas que participan en el estudio.

Como se observa en la figura 1, la mayoría de individuos de la muestra dirige escuelas de primaria, seguido de colegios y por último preescolar.

En cuanto a las jornadas laborales del colectivo de directores, $88,8 \%$ indica diurnas, mientras el restante $11,2 \%$ nocturnas. Igualmente, $72 \%$ (90) señala tener el puesto en propiedad y solamente $28 \%$ (35) es interino.

A nivel nacional, el Ministerio de Educación Pública determina el tipo de centro por la cantidad de estudiantes matriculados según el nivel de formación (Tabla 3), la cual puede variar desde la dirección tipo uno con menor número de estudiantes, hasta el tipo cinco con mayor cantidad de matrícula. En

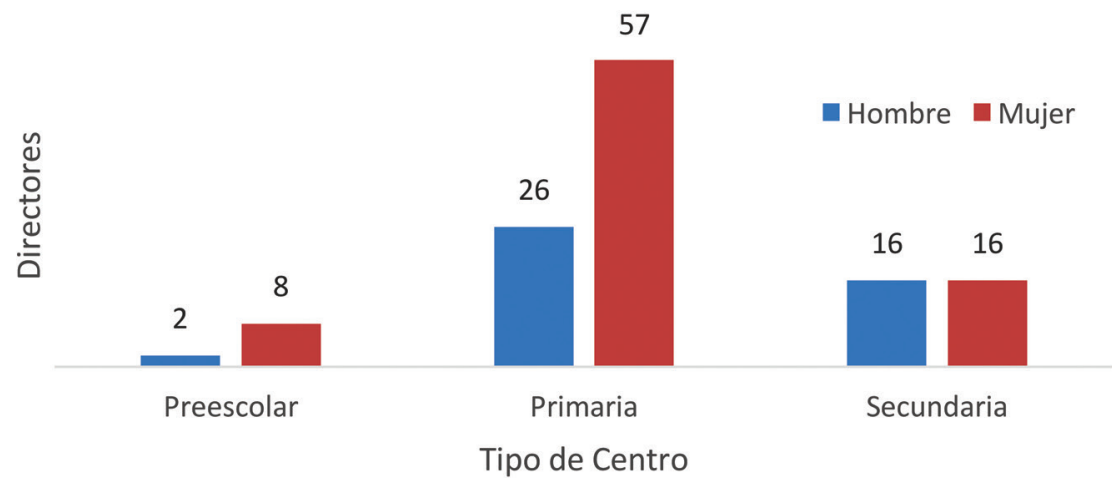

Figura 1. Distribución de centros educativos y sexo. Fuente: Elaboración propia. 
TABLA 3

Distribuciones de tipo de dirección por cantidad de estudiantes según centro educativo

\begin{tabular}{lccccc}
\multicolumn{1}{r}{ Centro educativo } & \multicolumn{3}{c}{ Tipo de dirección } \\
& Uno & Dos & Tres & Cuatro \\
Preescolar & Hasta 89 & $90-199$ & Más de 200 & - & Cinco \\
Primaria & $31-90$ & $91-200$ & $201-400$ & $401-800$ & Más de 800 \\
Secundaria & Hasta 500 & $501-1000$ & Más de 1000 & - & - \\
\hline
\end{tabular}

Fuente: Elaboración propia con datos de Resolución MEP (MEP-558-2013).

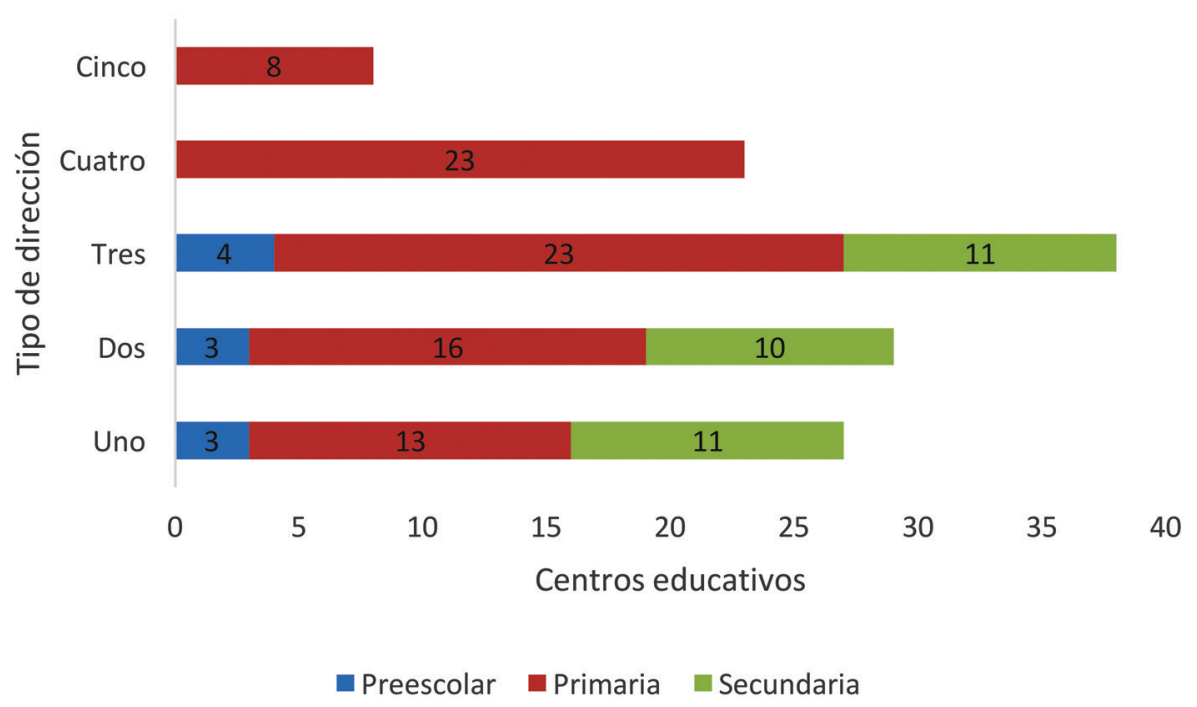

Figura 2. Distribución de centros educativos según tipo de dirección. Fuente: Elaboración propia.

la figura 2 se observa como tan solo $6,4 \%$ responde a una dirección tipo cinco, mientras que la de mayor frecuencia es de tipo tres $(28,8 \%)$, el resto de direcciones están en torno a $20 \%$.

A continuación, se presentan los resultados para cada uno de los tipos de liderazgo, así como para los tres componentes presentes en el instrumento derivados de la teoría.

En la tabla 4, se observan las frecuencias relativas de las preguntas que conforman la subescala de liderazgo transformacional. Entre las afirmaciones mejor valoradas se encuentra el ítem 36 "manifiesto mi confianza de que se alcanzarán los objetivos" ( $X=4,86 / \mathrm{DS}$ : 0,37$)$, seguido del ítem 13 "hablo con entusiasmo de las cosas que deben llevarse a cabo" ( $X=4,71 / D S=0,80)$, ambos pertenecientes al componente de motivación por inspiración. Igualmente, el ítem 31 "ayudo a los demás a desarrollar sus puntos fuertes" $(X=4,66 / D S=0,62)$ perteneciente al componente de consideraciones individuales, y el ítem 14 "hago hincapié en la importancia de tener una sólida visión de propósito" ( $X=4,65 / D S=0,71)$ del componente influencia idealizada.

En cuanto a las puntuaciones más bajas, se han dado en las afirmaciones 34 "recalco la importancia de tener un sentido colectivo de misión" ( $X=2,23 / \mathrm{DS}=1,26)$; el ítem $25(X=2,88 / \mathrm{DS}=1,53$ "hago ostentación de un sentido de poder y de confianza", seguido de los ítems $6(X=2,23 / D S=1,26)$ "hablo sobre mis creencias y valores más importantes", todos pertenecientes al componente influencia idealizada. 
TABLA 4

Porcentajes de frecuencia y estadísticos de la dimensión liderazgo transformacional

\begin{tabular}{|c|c|c|c|c|c|c|c|}
\hline Estimulación intelectual & 1 & 2 & 3 & 4 & 5 & $X$ & DS \\
\hline Reexamino asunciones de manera crítica para plantearme si son las adecuadas. & 4,0 & 1,6 & 16,8 & 32,0 & 45,6 & 4,13 & 1,05 \\
\hline Al resolver problemas busco diferentes perspectivas & 2,4 & 1,6 & 2,4 & 20,8 & 72,8 & 4,60 & 0,82 \\
\hline Hago que los demás enfoquen los problemas desde muchos ángulos distintos. & 4,0 & 2,4 & 8,0 & 40,8 & 44,8 & 4,20 & 0,98 \\
\hline Sugiero nuevas formas de considerar la realización de las tareas. & 0,8 & 0,8 & 5,6 & 33,6 & 59,2 & 4,50 & 0,71 \\
\hline \multicolumn{8}{|l|}{ Influencia idealizada (conducta) } \\
\hline Hablo sobre mis creencias y valores más importantes & 12,8 & 12,0 & 21,6 & 32,8 & 20,8 & 3,37 & 1,29 \\
\hline Hago hincapié en la importancia de poseer una sólida visión de propósito. & 1,6 & 0,0 & 4,0 & 20,8 & 73,6 & 4,65 & 0,71 \\
\hline Considero las consecuencias morales y éticas de mis decisiones. & 0,8 & 0,8 & 8,0 & 23,2 & 67,2 & 4,55 & 0,74 \\
\hline Recalco la importancia de tener un sentido colectivo de misión. & 36,8 & 31,2 & 9,6 & 16,8 & 5,6 & 2,23 & 1,26 \\
\hline \multicolumn{8}{|l|}{ Influencia idealizada (atributos) } \\
\hline Genero orgullo en los demás por relacionarse conmigo. & 20,8 & 7,2 & 16,8 & 20,8 & 34,4 & 4,12 & 1,08 \\
\hline Antepongo el interés del grupo, al mío propio. & 12,8 & 7,2 & 9,6 & 25,6 & 44,8 & 3,82 & 1,40 \\
\hline Mi modo de actuar provoca que los demás sientan respeto hacia mí. & 4,8 & 3,2 & 4,8 & 27,2 & 60,0 & 4,34 & 1,05 \\
\hline Hago ostentación de un sentido de poder y de confianza. & 29,6 & 13,6 & 16,0 & 20,8 & 20,0 & 2,88 & 1,53 \\
\hline \multicolumn{8}{|l|}{ Motivación por inspiración } \\
\hline Soy optimista cuando hablo del futuro. & 1,6 & 0,0 & 4,0 & 24,0 & 70,4 & 4,62 & 0,72 \\
\hline Hablo con entusiasmo de las cosas que deben llevarse a cabo. & 3,2 & 0,8 & 0,0 & 13,6 & 82,4 & 4,71 & 0,80 \\
\hline Transmito una visión convincente del futuro. & 2,4 & 0,8 & 8,8 & 33,6 & 54,4 & 4,37 & 0,87 \\
\hline Manifiesto mi confianza de que se alcanzarán los objetivos. & 0,0 & 0,0 & 0,8 & 12,8 & 86,4 & 4,86 & 0,37 \\
\hline \multicolumn{8}{|l|}{ Consideraciones individuales } \\
\hline Dedico tiempo a enseñar y a instruir. & 0,0 & 0,8 & 7,2 & 28,0 & 64,0 & 4,55 & 0,66 \\
\hline Trato a los demás cómo individuos más que como miembros del grupo. & 17,6 & 8,0 & 11,2 & 22,4 & 40,8 & 3,61 & 1,51 \\
\hline $\begin{array}{l}\text { Considero que cada individuo posee necesidades, aptitudes } \\
\text { y aspiraciones distintas a los demás. }\end{array}$ & 6,4 & 0,0 & 4,8 & 11,2 & 77,6 & 4,54 & 1,06 \\
\hline Ayudo a los demás a desarrollar sus puntos fuertes. & 0,0 & 0,8 & 5,6 & 20,8 & 72,8 & 4,66 & 0,62 \\
\hline
\end{tabular}

Fuente: Elaboración propia.

Los ítems mejor valorados de la dimensión liderazgo transaccional (Tabla 5) pertenecen a la subescala premio por contingencia, concretamente la afirmación $35(X=4,81 / D S=0,43)$ "expreso satisfacción cuando se cumplen las expectativas de los demás" y la $16(X=4,66 / D S=0,64)$ "dejo muy claro lo que uno puede esperar cuando se alcanzar los objetivos". Otra de los mayores promedios lo obtiene el ítem 4 ( $X=$ $4,54 / \mathrm{DS}=0,86$ ) "presto atención a las irregularidades, los errores, las excepciones y las desviaciones de la norma" correspondiente a la sub-escala gerencia por excepción activa.

En cuanto a las puntuaciones más bajas, cabe destacar las afirmaciones $12(X=1,58 / D S=1,12)$ "espero a que las cosas vayan mal antes de tomar medidas"; la $20(X=1,66 / D S=1,28)$ "considero que los problemas deben llegar a ser crónicos para tomar medidas" y la 17 ( $X=2,16$ / DS=1,35) "creo profundamente en la frase "si no está roto, no lo arregles", todos ellos pertenecientes a la subescala de gerencia por excepción pasiva.

En cuanto al estilo de liderazgo leizze-faire (Tabla 6), destacan las bajas puntuaciones obtenidas en todas las afirmaciones, por ejemplo, el ítem $28(X=1,55, D S=1,07)$ "evito tomar decisiones" o el ítem $7(X=1,70$, $\mathrm{DS}=1,33)$ "me ausento cuando se me necesita".

Por último, como se observa en la tabla 7, en relación a las subescalas de efectividad, esfuerzo extra y satisfacción, cabe destacar las altas puntuaciones en los tres aspectos medidos, donde los promedios 
TABLA 5

Porcentajes y estadísticos de la dimensión liderazgo transaccional

\section{Premio por contingencia}

Ofrezco ayuda a los demás a cambio de sus esfuerzos.

Discuto detenidamente quién es el responsable de alcanzar los objetivos de rendimiento.

Dejo muy claro lo que uno puede esperar cuando se alcanzan los objetivos. Expreso satisfacción cuando se cumplen las expectativas de los demás.

\section{Gerencia por excepción pasiva}

No intervengo a no ser que los problemas presenten gravedad.

Espero a que las cosas vayan mal antes de tomar medidas.

Creo profundamente en la frase "Si no está roto, no lo arregles".

Considero que los problemas deben llegar a ser crónicos para tomar medidas.

\section{Gerencia por excepción activa}

Presto atención a las irregularidades, los errores, las excepciones y las desviaciones de la norma.

Centro toda mi atención en resolver los errores, las quejas y los fallos que se producen.

Sigo con atención todos los errores.

Dirijo mi atención hacia los casos que no cumplen las normas.

$\begin{array}{ccccccc}1 & 2 & 3 & 4 & 5 & X & \text { DS } \\ 24,8 & 9,6 & 9,6 & 20,0 & 36,0 & 3,33 & 1,62 \\ 4,0 & 5,6 & 12,0 & 31,2 & 47,2 & 4,12 & 1,08 \\ & & & & & & \\ 0,8 & 0,0 & 4,0 & 23,2 & 72,0 & 4,66 & 0,64 \\ 0,0 & 0,0 & 1,6 & 16,0 & 82,4 & 4,81 & 0,43 \\ & & & & & & \\ 16,0 & 14,4 & 15,2 & 37,6 & 16,8 & 3,25 & 1,34 \\ 70,4 & 16,8 & 1,6 & 6,4 & 4,8 & 1,58 & 1,12 \\ 44,0 & 25,6 & 11,2 & 8,8 & 10,4 & 2,16 & 1,35 \\ 71,2 & 13,6 & 2,4 & 3,2 & 9,6 & 1,66 & 1,28\end{array}$

$\begin{array}{lllllll}1,6 & 1,6 & 9,6 & 15,2 & 72,0 & 4,54 & 0,86\end{array}$

$\begin{array}{lllllll}0,8 & 4,0 & 20,8 & 26,8 & 48,0 & 4,17 & 0,95\end{array}$

$3,2 \quad 1,6 \quad 8,8 \quad 33,6 \quad 52,8 \quad 4,31 \quad 0,94$

$\begin{array}{lllllll}7,2 & 6,4 & 19,2 & 31,2 & 36,0 & 3,82 & 1,20\end{array}$

Fuente: Elaboración propia.

TABLA 6

Porcentajes y estadísticos de la dimensión liderazgo leizze faire

\begin{tabular}{|c|c|c|c|c|c|c|c|}
\hline Leizze-faire & 1 & 2 & 3 & 4 & 5 & $\mathrm{X}$ & DS \\
\hline Evito involucrarme cuando surgen cuestiones de importancia. & 53,6 & 14,4 & 8,8 & 14,4 & 8,8 & 2,10 & 1,41 \\
\hline Me ausento cuando se me necesita. & 72,8 & 8,8 & 3,2 & 5,6 & 9,6 & 1,70 & 1,33 \\
\hline Evito tomar decisiones. & 72,0 & 15,2 & 1,6 & 8,0 & 3,2 & 1,55 & 1,07 \\
\hline Me retraso en dar respuesta a cuestiones urgentes. & 36,8 & 31,2 & 9,6 & 16,8 & 5,6 & 2,23 & 1,26 \\
\hline
\end{tabular}

Fuente: Elaboración propia.

TABLA 7

Porcentajes y estadísticos de las subescalas efectividad, esfuerzo extra y satisfacción

\section{Efectividad}

Soy eficaz en satisfacer las necesidades de índole laboral de los demás.

Soy eficaz al representar a mi grupo ante autoridades superiores.

Soy eficaz en satisfacer los requisitos del centro educativo.

Lidero un grupo que es eficaz.

\section{Esfuerzo extra}

Hago que los demás hagan más de lo que esperaban hacer.

Realzo en los demás el deseo de triunfar.

Aumento la voluntad de los demás para poner el máximo desempeño.

\section{Satisfacción}

Utilizo métodos de liderazgo agradable.

Trabajo satisfactoriamente con los demás.

Fuente: Elaboración propia. 
TABLA 8

Estadísticos descriptivos de los componentes del MLQ 5X

\begin{tabular}{lcccc}
\multicolumn{1}{c}{ Cuestionario MLQ 5X } & Mínimo & Máximo & Media & Desv. típ. \\
Estimulación intelectual & 2,25 & 5,00 & 4,35 & 4,57 \\
Influencia Ide. conducta & 2,75 & 5,00 & 3,61 & 0,50 \\
Influencia Ide. atributos & 1,25 & 5,00 & 4,63 & 4,78 \\
Motivación por inspiración & 2,50 & 5,00 & 4,33 & 0,46 \\
Consideraciones individuales & 2,75 & 5,00 & 4,22 & 0,57 \\
Premio por contingencia & 3,00 & 5,00 & 4,16 & 0,54 \\
Gerencia exc. pasiva & 1,00 & 4,25 & 4,21 & 0,61 \\
Gerencia exc. activa & 2,50 & 5,00 & 4,89 & 0,82 \\
Laissez faire & 1,00 & 5,00 & 4,49 & 0,56 \\
Esfuerzo extra & 2,00 & 5,00 & 4,64 & 0,39 \\
Efectividad & 3,25 & 5,00 & 5,00 & \\
Satisfacción & 3,00 & &
\end{tabular}

Fuente: Elaboración propia.

obtenidos en el total de afirmaciones oscilan en el rango de $X=4,07$ y $X=4,78$, con desviaciones típicas relativamente bajas, que indican poca variabilidad de los datos.

Como se observa en la tabla 8, todos los componentes del liderazgo transformacional obtienen promedios muy altos, siendo el tipo de liderazgo predominante de la escala aplicada, seguido del liderazgo transaccional con promedios ligeramente más bajos, destacando que la baja puntuación en la subescala gerencia por excepción pasiva es debido a la redacción negativa de los ítems, aspecto que debe tenerse en cuenta a la hora de realizar el análisis correspondiente.

En el caso del estilo de liderazgo laissez-faire, se denota como la puntuación es la más baja de la escala $(X=1,89 / \mathrm{SD}=0,82)$, indicando la poca presencia de este tipo de liderazgo en la muestra encuestada. Igualmente, y como se ha comentado con anterioridad, las subescalas esfuerzo extra, efectividad y satisfacción, obtienen puntuaciones sumamente altas, lo que indica presencia de estos aspectos en los estilos de liderazgo del colectivo director.

En la figura 3, se observa cómo 93,6\% tiene presencia alta del estilo de liderazgo transformacional y solamente $6,4 \%$ restante se ubica en presencia media. En cuanto al estilo transaccional, solamente $27,2 \%$ se ubica en una categoría media, mientras la gran mayoría $(72,8)$ en categoría alta. Cabe destacar la baja presencia $(74,4 \%)$ del estilo laissez-faire, media en $21,6 \%$ y solamente $4,0 \%$ alta. Por último, la subescala satisfacción y esfuerzo extra, muestran mayor presencia con porcentajes de 97,6\% y 90,45 respectivamente, mientras la satisfacción tiene presencia media, ubicando a 99,2\% en esta categoría y tan solo $0,8 \%$ en la categoría baja. 


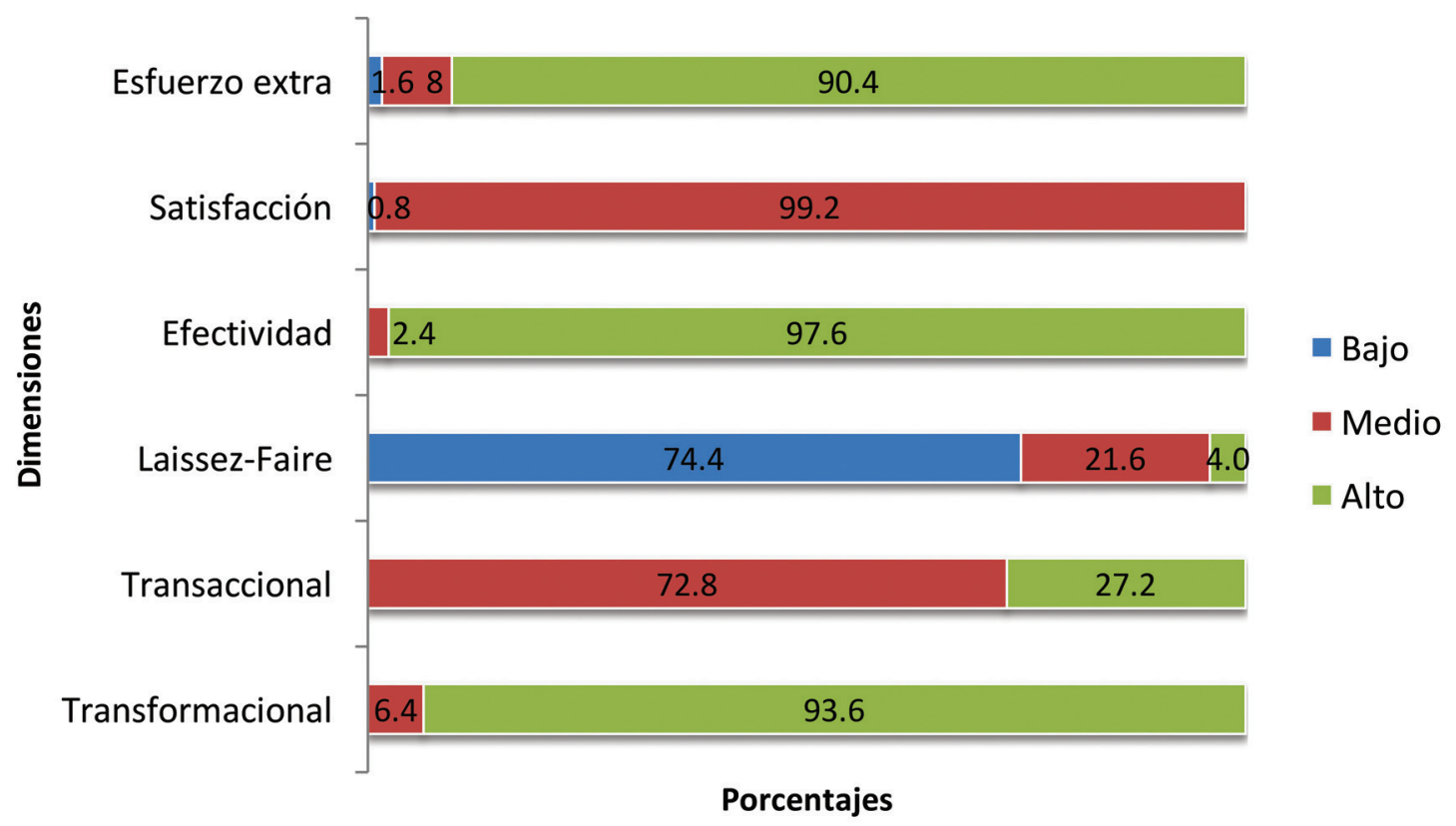

Figura3. Distribución de frecuencias relativas según tipo de liderazgo. Fuente: Elaboración propia.

\section{DISCUSIÓN}

En relación con el liderazgo transformacional, el componente estimulación intelectual obtiene altas puntuaciones en la escala, lo cual se asocia principalmente a la resolución de problemas desde diferentes perspectivas, esto a su vez indica la presencia de espacios de diálogo en la gestión del centro. Lo anterior es una de las características más destacadas para el trabajo colaborativo y sumamente necesario para gestionar procesos de mejora vinculados a la calidad de la oferta educativa (Avolio \& Bass, 2004).

La persona directiva juega un papel de liderazgo para trocar los intereses, características y necesidades individuales en esfuerzos colectivos. Dicha condición es uno de los desafíos más grandes con que se enfrentan los directivos en la gestión de los centros educativos costarricenses, ya que la selección del personal no es de vinculación directa en las funciones que ejerce (Hermosilla et al., 2016).

En cuanto a la influencia idealizada, se obtienen puntuaciones muy altas, que quedan relacionadas a la conducta del directivo y enfatizan en la importancia de una visión de los propósitos institucionales, en concordancia con una labor pensada, planificada y estructurada para favorecer la participación de sus miembros.

Lo anterior permite no solo articular esfuerzos conjuntos entre la gestión del centro y el cuerpo docente, sino que también incentiva la imagen del directivo como líder y sirve como fuente de inspiración para sus colaboradores, ya que la coherencia en la conducción del centro educativo, junto con la visión que se estable en conjunto con el equipo docente, permite el logro de objetivos de manera más eficiente, aspecto vinculado con lo expuesto por Molero et al., (2010). Desde esta perspectiva el rol de la persona directiva conlleva una carga emocional, ya que sus colaboradores lo asumen en muchas ocasiones como modelo por seguir.

Sin embargo, se observa en este componente una separación entre la misión y la visión del centro, en el sentido de que la primera obtiene puntuaciones medias bajas $(2,23)$ mientras la segunda obtiene puntuaciones altas $(4,65)$, aspecto que debilita el liderazgo transformacional, ya que la misión favorece un 
sentimiento de pertenencia y emocionalidad hacia la labor de la institución, mientras la visión, un sentimiento de orgullo y claridad en la ruta trazada para el logro de metas. Por lo tanto, si los directivos están abocados a cultivar una visión sólida del propósito del centro educativo, también deberían incentivar un sentido de misión colectiva que propicie los procesos identitarios para alcanzar un equipo que trabaje desde espacios de colaboración mutua, en comunión con lo indicado por Leithwood (2009).

El papel de la persona directiva para la construcción colectiva de la misión y la visión del centro es muy importante, ya que trasciende el cumplimiento administrativo, para convertirse en un sello distintivo de la institución y todo su quehacer.

En la misma línea de análisis de la influencia idealizada ligada a los atributos, componente con mayores puntuaciones del estilo de liderazgo transformacional, se establece que los directivos perciben que su modo de actuar como gestores del centro educativo genera respeto entre sus colaboradores, condición relevante para quien lidera la institución.

Cabe destacar que el líder no ostenta su poder por la investidura del puesto, sino todo lo contrario, su legitimación como líder se deriva de su coherencia en el accionar desde marcos éticos en la toma de decisiones, de acuerdo con Montero et al. (2017). En este sentido la legitimación de la comunidad educativa trasciende el cargo, condición que se vuelve de elemental comprensión para las personas que ostentan puestos directivos.

Siguiendo con los aspectos relacionados con el liderazgo transformacional se establece la motivación por inspiración, igualmente con altas puntuaciones obtenidas, en el cual los directivos consultados desvelaron que una de las claves en su gestión es manifestar confianza a sus colaboradores para el logro de objetivos, aspecto vinculado a la capacidad del directivo escolar de trascender sus propios intereses por los de la organización, uno de los elementos clave para el desarrollo de una imagen de autoridad moral y ética aceptable en el centro educativo, de acuerdo con Hermosilla et al. (2016). Igualmente, se relaciona con los deseos de superación y éxito de sus docentes hacia el trabajo compartido y coincide con lo indicado por Garbanzo y Orozco (2007).

Continuando el análisis del liderazgo transformacional, el componente de consideraciones individuales valorado por los directivos, coincide con las consideraciones planteadas por Stilman (2001), Mendoza y Ortiz (2006) y Alatorre (2016), ya que los resultados muestran que ayudan a los miembros del equipo de trabajo a desarrollar sus puntos fuertes, condición que genera un ambiente de crecimiento permanente como parte de la cultura organizacional.

Por otro lado, se establece entre los directivos participantes que los premios por contingencia los ejecutan expresando satisfacción a sus colaboradores cuando cumplen sus expectativas. Esta práctica refuerza el trabajo en equipo, mientras el directivo asume la responsabilidad de dar seguimiento a las tareas asignadas y a la vez incentiva el logro de las metas, lo cual coincide con lo planteado por Contreras y Barbosa (2013). Sin embargo, la práctica requiere de honestidad por parte de la persona directiva, para no caer en acciones que generen conflictos entre los miembros del equipo y competencias desleales que pueden percibirse como acciones excluyentes por parte de otros colaboradores.

Otro componente del liderazgo transaccional es la gerencia por excepción pasiva, con puntuaciones bajas, donde los directivos expresan que no intervienen hasta que los problemas son graves, aspecto que puede visualizarse desde una doble vertiente, por un lado, genera la posibilidad de que el cuerpo docente y administrativo sean capaces de resolver sus propios conflictos; por otro, puede ser arriesgado para la dirección intervenir cuando los problemas adquieren demasiada envergadura. Esta práctica asumida de manera recurrente en los centros educativos puede generar más aspectos negativos que positivos para sus colaboradores. 
En cuanto a la gerencia por excepción activa, los resultados muestran puntuaciones elevadas en los ítemes, los cuales derivan en atención a las irregularidades, los errores, las excepciones y las desviaciones de la norma, situación que incentiva un clima punitivo vinculado a una cultura organizacional apegada a los reglamentos, restringiendo la capacidad creativa de sus miembros, lo cual coincide con lo indicado por Stilman (2001), Mendoza y Ortiz (2006) y Alatorre (2016).

En cuanto al estilo de liderazgo laizze-faire, cabe destacar las bajas puntuaciones obtenidas, es decir que es el de menor predominancia en los directivos que participaron en la investigación, aspecto positivo desde este análisis. Destaca, sin embargo, con el puntaje más alto de la subescala el retraso en dar respuestas a cuestiones urgentes, esto se asocia a la carga de tareas y funciones que asumen las personas que figuran en cargos de dirección escolar. Lo anterior concuerda con lo indicado por Vílchez et al. (2019) al proponer que en este estilo de liderazgo exista una mínima vinculación del directivo con los procesos de gestión del centro educativo.

La investigación también contempla tres variables adicionales que son la efectividad, el esfuerzo extra y la satisfacción como parte complementaria de la indagación, se resaltan las altas puntuaciones obtenidas y que derivan en la integración de estas variables en sus prácticas de liderazgo, lo cual representa un aspecto positivo en el tanto el directivo genera procesos identitarios en sus funciones, así como en el logro de metas colectivas trazadas, mientras contribuye en una visión integral de la organización que concuerda con lo expuesto por Bass y Avolio (1994) y Macías y Ramírez (2015).

\section{CONCLUSIONES}

Como se observa en los resultados, el estilo de liderazgo predominante en los directivos de los centros educativos de la provincia de Heredia (Costa Rica) es el transformacional, seguido del transaccional y por último destaca la poca predominancia del estilo laissez-faire.

Se destacan en el liderazgo transformacional los espacios de diálogo y reflexión que cimientan un trabajo colaborativo, el cual favorece la participación de sus miembros en propuestas de solución para las necesidades que les aquejan. Igualmente, se promueven procesos identitarios requeridos para una cultura institucional con miras a una educación de calidad.

El rol de la persona directiva se plantea en términos de liderazgo participativo que promueva una organización dinámica, flexible, con claridad de su misión y visión y el papel que juega como transformador social.

La legitimación de la persona directiva lo brinda la comunidad educativa, por tanto, la aceptación del modo de actuar bajo marcos éticos, destaca como un elemento relevante en la imagen que se construye desde el colectivo hacia la dirección.

Los directivos de la DRH se caracterizan igualmente por un liderazgo transaccional, el cual se destaca por procesos planificados, dirigidos al logro de metas y objetivos en los centros educativos. Las personas directivas asumen un rol práctico de asignación de tareas y seguimiento puntal para lo establecido, lo que genera en los miembros de la comunidad educativa credibilidad en la consecución de metas. Sin embargo, esta condición no es sinónimo de trabajo colaborativo, ya que la designación de tareas u objetivos puede plantearse de manera individualizada, sin contribuir a la transformación requerida para una educación de calidad.

Cabe destacar la poca predominancia del estilo laissez-faire en las personas directivas. Aspecto positivo, ya que no se vincula a procesos de transformación social, sino al rol centrado en la tramitología administrativa, la cual sostiene el statu quo, pero no hace aportes para brindar una oferta educativa de calidad. 
Es importante destacar que los estilos de liderazgo se vinculan con el éxito de los centros educativos, por ello la promoción del trabajo colaborativo, al trazar metas conjuntas, se asocia con una oferta de calidad en tanto responda a las necesidades e intereses del contexto educativo.

Finalmente, se destaca que la dimensión pedagógica es relevante para cualquier estilo de liderazgo vinculado a procesos de formación. Es necesario destacar que las personas con un cargo de dirección deberán mostrar claridad sobre su papel como agentes de cambio y transformación social.

\section{REFERENCIAS}

Alatorre, A. (2016). Interdependencia entre el liderazgo transformacional, cultura organizacional y cambio educativo: una reflexión. REICE. Revista Iberoamericana sobre Calidad, Eficacia y Cambio en Educación, 11(1). Recuperado de https://revistas.uam.es/index.php/reice/article/view/2915

Allen, N., Grigsby, B. \& Peters, M. (2015). Does Leadership Matter? Examining the Relationship among Transformational Leadership, School Climate, and Student Achievement. International Journal of Educational Leadership Preparation, 10(2), 1-22. Recuperado de https://eric.ed.gov/?id=EJ1083099

Antonankis, J., Avolio, B. \& Sivasubramaniam, N. (2003). Context and leadership: An examination of the nine-factor full-range leadership theory using the Multifactor Leadership Questionnaire. Leadership Quarterly, Vol. 14, pp. 261-295. Retrieved from https://www.sciencedirect.com/science/article/pii/ S1048984303000304

Arnal, J., Rincón, D. \& Latorre, A. (1992). Investigación educativa: fundamentos y metodología. Labor.

Avolio, B., Bass, B. \& Jung, D. (1999). Re-examining the components of transformational and transactional leadership using the Multifactor Leadership Questionnaire. Journal of Occupational and Organizational Psychology, Vol. 72, pp. 441-462. DOI: https://doi.org/10.1348/096317999166789

Avolio, B. \& Bass, B. (2004). Multifactor Leadership Questionnaire (3a ed.). Palo Alto: Mind Garden.

Bagheri, R., \& Sohrabi, Z. (2015). Psychometric properties of Persian version of the multifactor leadership questionnaire (MLQ). Medical journal of the Islamic Republic of Iran, 29, 256. Recuperado de https:// www.ncbi.nlm.nih.gov/pmc/articles/PMC4715420/

Bass, B. \& Avolio, B. (Eds.). (1994). Improving organizational effectiveness through transformational leadership. Sage.

Botía, A. (2015). Un liderazgo pedagógico en una comunidad que aprende. Padres y maestros/Journal of Parents and Teachers, (361), 23-27. Recuperado de http://revistas.upcomillas.es/index.php/ padresymaestros/article/view/5338

Botía, A., Rodríguez, K. \& García-Garnica, M. (2017). Evaluación multidimensional del liderazgo pedagógico: claves para la mejora escolar. Ensaio: Avaliação e Políticas Públicas em Educação, 25(95), 483-506. Recuperado de http://www.scielo.br/scielo. php?pid=S010440362017005001105\&script=sci_abstract\&tlng=pt

Bryant, P., Butcher, J. \& O'Connor, J. (2016). Improving School Leadership: The Connection of Transformational Leadership and Psychological Well-Being of the Followers. School Leadership Review, 11(2), 6. Recuperado de https://scholarworks.sfasu.edu/str/vol11/iss2/6/

Buitrago, J., Escobar, J. \& Terraza, L. (2017). Los grupos informales en las organizaciones escolares: un acercamiento desde la gestión educativa. Praxis, 56-68. Recuperado de http://revistas.unimagdalena.edu.co/index.php/praxis/article/view/2068

Cerdas, V., Torres, N. \& García, J.A. (2016). Tendencias y desafíos de la Administración Educativa costarricense: la génesis de un proyecto. Revista Gestión de la Educación. I. 6, №2, (119-136). DOI: http://dx.doi. org/10.15517/rge.v1i2.25495 
Contreras F. \& Barbosa D. (2013). Del liderazgo transaccional al liderazgo transformacional: implicaciones para el cambio organizacional. Revista Virtual Universidad Católica del Norte, núm. 39, mayo-agosto, 2013, pp. 152-164 Fundación Universitaria Católica del Norte Medellín, Colombia. Recuperado: http://www.redalyc.org/pdf/1942/194227509013.pdf

Corbella, M. (2016). Liderazgo y responsabilidad educativa: el necesario liderazgo de directores y profesores en la educación. Revista Fuentes, (14), 85-104. Recuperado de https://revistascientificas.us.es/ index.php/fuentes/article/view/2354

Delgado, M. (2005). El liderazgo en las organizaciones educativas: revisión y perspectivas actuales. Revista española de pedagogía, 367-388.Recuperado https://www.jstor.org/ stable/23766325?seq=1\#page_scan_tab_contents

Garbanzo, G., \& Orozco, V. (2007). Desafíos del sistema educativo costarricense: un nuevo paradigma de la administración de la educación. Educación, 31(2). Recuperado de http://www.redalyc.org/ html/440/44031207/

García-Solarte, M. (2015). Formulación de un modelo de liderazgo desde las teorías organizacionales. Entramado, 11(1), 60-79. Recuperado de https://dialnet.unirioja.es/servlet/articulo?codigo=5473633

García, J., Cerdas, V. \& Torres, N. (2018). Gestión curricular en centros educativos costarricenses: Un análisis desde la percepción docente y la dirección. Revista Electrónica Educare, 22(1), 8. Recuperado de https://dialnet.unirioja.es/servlet/articulo?codigo $=6228636$

Hermosilla, D., Amutio, A., Costa, S., \& Páez, D. (2016). El Liderazgo transformacional en las organizaciones: variables mediadoras y consecuencias a largo plazo. Revista de Psicología del Trabajo y de las Organizaciones, 32(3), 135-143. DOI: http://dx.doi.org/10.1016/j.rpto.2016.06.003

Hernández, J., Tobón, S., \& Vázquez, J. (2015). Estudio del liderazgo socioformativo mediante la cartografía conceptual. RIEE. Revista Iberoamericana de Evaluación Educativa. Recuperado de https:// repositorio.uam.es/handle/10486/670648

Hernández, R., Fernández, C. \& Baptista, P. (2010). Metodología de la investigación. México D.F., México: Editorial Mc Graw Hill.

Horn, A. \& Marfán, J. (2010). Relación entre liderazgo educativo y desempeño escolar: Revisión de la investigación en Chile. Psicoperspectivas, 9(2), 82-104. Doi: http://dx.doi.org/10.5027/ psicoperspectivas-Vol9-Issue2-fulltext-116

Leithwood, K. (2009). ¿Cómo liderar nuestras escuelas? Aportes desde la investigación. Inscripción registro de propiedad intelectual 184.085 / ISBN: 978-956-8200-18-3 Traducción: Cristóbal Santa Cruz. Santiago. Chile.

Loaiza, C. (2017). Liderazgo Organizacional y Capital Humano. Revista Venezolana de Gerencia, 22(77), 5-7. Recuperado de https://www.redalyc.org/pdf/290/29051457001.pdf

Macías, R. \& Ramírez, A. (2015). Liderazgo educativo transformacional como necesidad de las instituciones educativas en la República de Ecuador. REFCalE: Revista Electrónica Formación y Calidad Educativa. ISSN 1390-9010, 3(2), 109-120. Recuprado de https://www.researchgate.net/publication/319621175_LIDERAZGO_EDUCATIVO_TRANSFORMACIONAL_COMO_NECESIDAD_DE_LAS_ INSTITUCIONES_EDUCATIVAS_EN_LA_REPUBLICA_DE_ECUADOR

Manning, J. (2016). The influence of nurse manager leadership style on staff nurse work engagement. Journal of Nursing Administration, 46(9), 438-443. Recuperado de https://www.ncbi.nlm.nih.gov/ pubmed/27496584

Marticorena, R. (2018). Liderazgo institucional en organizaciones educativas. Recuperado de http://repositorio.uigv.edu.pe/handle/20.500.11818/3086

Mendoza, M. \& Ortiz, C. (2006). El liderazgo transformacional, dimensiones e impacto en la cultura organizacional y eficacia de las empresas. Revista Facultad de Ciencias Económicas: Investigación y Reflexión, 14(1). Recuperado de http://www.redalyc.org/html/909/90900107/ 
Molero, F., Recio, P. \& Cuadrado, I. (2010). Liderazgo transformacional y liderazgo transaccional: un análisis de la estructura factorial del Multifactor Leadership Questionnaire (MLQ) en una muestra española. Psicothema, vol. 22, núm. 3, pp. 495-501.Universidad de Oviedo, España. Recuperado de https://www.redalyc.org/html/727/72714400022/

Montero, E., Fernández, A., Barquero, K. \& Martínez, L. (2017). ¿Es "real" el descenso en los puntajes de Costa Rica en las pruebas PISA 2015? Sexto Informe Estado de la Educacion. Recuperado de http://www.estadonacion.or.cr/files/biblioteca_virtual/educacion/006/primaria-y-secundaria/ Montero_E.pdf

Programa Estado de la Nación. (2017). Sexto informe estado de la educación / PEN. 1 ed. San José C.R: Servicios Gráficos, A. C. ISBN-978-9930-540-02-2

Ruiz, M. (2013). Liderazgo y responsabilidad educativa. Revista Fuentes, 14; pp. 85-104. Universidad Nacional de Educación a Distancia. Recuperado de: https://ojs.publius.us.es/ojs/index.php/ fuentes/article/view/2354/2173

Sánchez, A. (2015). Importancia e impacto del liderazgo educativo. Padres y Maestros/Journal of Parents and Teachers, (361), 6-11. Recuperado de http://revistas.upcomillas.es/index.php/padresymaestros/ article/view/5335

Stilman, F. (2001). Liderazgo y cultura organizacional. Buenos Aires: Universidad de Buenos Aires. Facultad de Ciencias Económicas. Escuela de Estudios de Posgrado. Recuperado de http://bibliotecadigital.econ.uba.ar/download/tpos/1502-0120_StilmanF.pdf

Torrecilla, F. (2016). La investigación sobre liderazgo educativo: una mirada desde el presente proyectada al futuro. Revista Fuentes, (14), 9-14. Recuperado de file:///D:/Documents/Downloads/2351-49351-PB.pdf

Trujillo, J., López, J. \& Lorenzo, M. (2016). Liderazgo educativo en la gestión de redes sociales en la web 2.0. Innovación y cambio en las organizaciones educativas. REICE. Revista lberoamericana sobre Calidad, Eficacia y Cambio en Educación, 7(3). Recuperado de https://revistas.uam.es/index.php/ reice/article/view/5400

Vílchez, J., Alberola, E., Monte, P.\&Ferraz, H. (2019). Relación entre los estilos de liderazgo Transformacional y laissez-faire y el Síndrome de Quemarse por el Trabajo en profesores de educación secundaria. Acciones e investigaciones sociales, (39), 223-254. Recuperado de https://dialnet.unirioja.es/servlet/ articulo?codigo $=6728778$

Zenteno, M. \& Álvarez, A. (2016). La transformación institucional fundamento para el logro de la Calidad. Atenas, 3(35), 157-172. Recuperado https://atenas.mes.edu.cu/index.php/atenas/article/ view/224/415 